\title{
Study of Social Network Site Interactivity to Identify and Avert Usability Flaws for Effective User's Experience
}

\author{
Sami Abduljalil, Gi-Hyun Hwang, and Dae-Ki Kang, Member, KIMICS
}

\begin{abstract}
Due to the wide growth and popularity of social network website, large numbers of users discover these social network sites are a place where they can be able to spend their leisure time sharing interests, sharing ideas freely, sharing personal experience, and also to search for new friends or partners. These websites give an opportunity for its users to socialize with new people and to keep in touch or reconnect with current or old friends and families across disperse continents, which traditionally replace the common traditional methods. These social network websites need accurate and careful investigations and findings on the usability issues for effective interactivity and more usability. However, little research might have previously invested on the usability of these social network websites. Therefore, we propose a new framework to study and test the usability of these social network sites. We namely call our framework "Interactivity". This framework will enable developers to assess the usability of the social network sites. It will provide an overview of the user's behavior while interacting in these social network websites. Performance of the framework will be performed using Camtasia software. This software will entirely capture the interactivity of users including the screen and the movements, which the screen and the motion of the user action will undergo to analysis at the end of our research.
\end{abstract}

Index Terms - Interactivity Framework, Social Network sites, Human Factors, Usability

\section{INTRODUCTION}

IN the recent years, varieties of business organizations have inclined toward electronic business, which becomes conventional way to many Internet users. These organizations have developed numerous of social and commercial web sites to advertise and sell their products and to offer their clients more benefits. However, these organizations find the demand and the inclination of user

Manuscript received April 18, 2011; revised May 16, 2011; accepted May 27, 2011.

Sami Abduljalil is a graduate student at Ubiquitous IT graduate school of Dongseo University, Busan, 617-716, Korea (Email: samialkindi0708 (a)gmail.com)

Gi-Hyun Hwang is with the Division of Computer and Information Engineering, Dongseo University University, Busan, 617-716, Korea (Email: hwanggh@gdsu.dongseo.ac.kr)

Dae-ki kang (corresponding author) is with the Division of Computer and Information Engineering, Dongseo University University, Busan, 617-716, Korea (Email: dkkang@dongseo.ac.kr) for online social network is getting world's attraction. Popularity of social network sites environments has widely increased, which it provides a vibrant platform for many usability and sociality researchers who are seeking to find answer for issues related to the sociality and usability [1]. In other words, we can deem that, the development environments in online social network site have dramatically increased in the recent years. The increase developments of online social network sites environments entail the effectiveness of these social network web sites to perceive user's satisfactions and attitude [2]. For example, Facebook facilitates the interaction between users, which allows individuals to articulate a list of other users with friend who share same connection and interest, allow individuals to share status, photos, and videos. Therefore, Facebook can enhance the relationship of the users in terms of construction of public and semi-public profiles and building even relationship with strangers. Despite the growing attention to online social network sites, little is underestimated in the social network site designs, which are the usability and the user's experience as well as the user's behavior in interacting with these social network sites. Therefore, many professional Web developer and Web designers have developed guidelines that work well for organization that have robust resources to implement extensive user studies and to hire specialist. In our research we use Interactivity framework to evaluate the usability of these social network sites, and to study the user behavior for the efficiency of user satisfactions. Interactivity framework is typically a mechanism of studying the interaction between users and systems. Interactivity provides an alignment with a mission and goals of the social network sites because they basically create a framework that is fair, communal, and freedom of communication.

\section{LITERATURE REVIEW}

\section{A. Human Factors}

The field of human-computer interaction in the recent times has revealed the increase interest of researchers and scientist in generating scientific and methodological theory and knowledge about the interactivity of design in any system for human. Since the development of many systems is increased dramatically, the human factors are greatly playing the role in the system or web site development environments. Generally, Human factor is 
concerned about the human's capability in interacting with computers. It involves the human's physical and mental abilities. These abilities are varying from one individual to another. In other words, it is concerned about the hardware or software design. Human Factors has its origins in the Industrial Revolution and emerged as a full-fledged discipline during World War II. It was recognized that aircraft cockpit design needed to consider the human interface for controls and displays. Design Engineers were focused on the technology while Industrial Psychologists worked to optimize the interface. In some cases, Human Factors design can affect bottomline profitability or can be a life and death matter, e.g., you don't want to push the wrong button or mistake meters for kilometers in a spacecraft. Companies came to realize that a products success is dependent upon good Human Factors design. Human Factors is often used interchangeably with User Interface Design or HumanComputer Interface. There is a lot of overlap in these disciplines; however, arguably, Human Factors generally refers to hardware design while HCI generally refers to software design. Engineering Psychologist work in both disciplines and the overlap is considered greater than the difference. Human factor is considerably specialized to reveal more usability and interfaces problems in HumanComputer-Interaction (HCI). Human factors have been studied from many perspectives crossing sundry category of applications. Professor Robert J. Stone examines the human factors that affect the interactivity of online social games [3].

Although, human factor is appropriately useful to offer a comprehensive range of web design, but, still regrettably, the development strategy of web design lack to the identification and implementation of these factors. The comprehensive discovery of these factors incorporately leads to a reasonable and satisfactory web design. Relatively, the interchange correlation between the human and web site is the interactivity. The high-level of interactivity in a web site, the high number of users are increased and vice versa. To avert the flaws and obstacles in the interactivity, a serious consideration should be taken into account. So, avoidance of problems in the interactivity can lead to success of the web design and attraction.

\section{B. Interactivity}

Interactivity is the mainstay of any web site successful usability. The interpersonal human communication is measured by the increase of the user autonomy, which user can have control over the interaction and the navigation between the site pages. Ultimately, interactivity means the user is freely able to obtain the thing they want with a minimal time and efforts and with the avoidance of external or internal destruction on the web. External destruction can be defined by loading a web site with ads and multimedia contents, which hamper user from performing the task successfully. Internal destruction can be referred to functionality. The significance of the interactivity of the web underlies the user-efficacy in having control over the contents of the web sites. The interactivity framework gives an exposure to new feature that web developer adheres to implement during the development of web sites. Figure 1 is a simple structure of user interacting with a web server. Generally speaking, accessing the web needs a user interface which obviously well know phenomena. The user interface is the main communication platform between the system and users. However, users interfaces vary according to a web designer believe and interest or experience. But designing interface either web interface or application interface in regard to what web designer think is appropriate may result in failure of meeting a user acceptance. Interface should meet the interchangeable correlation of the clarity of interactivity and the user experience. Interactivity is an essential element, which it should be saliently emerged on the web design.

Recently, interactivity is a key component of Web site success. However, interactivity is the focal point of the website which addresses the following issues:

- How to motivate a user to stay longer than the expectation on the website.

- How to let the user recommends the website to their friends or partners.

- How to let the user frequently visits the website.

- How to let the users have self-autonomy during the interaction.

The aforementioned issues should be addressed in the design stages. These issues can derive new features and ways of interactivity. To support the implementation of the interactivity issues, the web designer and developer needs to adhere and adopt new strategy to advance the interaction. The new strategy needs to be in compliance with the web goals.

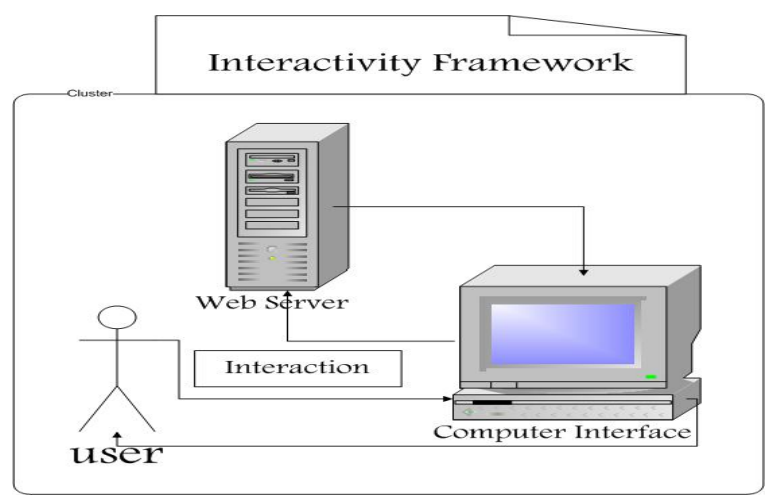

Fig. 1. Structure of the interaction between users and system. 
Furthermore, interactivity has been the center-focus of many scholar interests. Ruby et al has suggested a theoretical framework to interactivity and revisit the websites [4]. He more concerns on how interactivity of a particular website can be able to frequently let the user revisit, which leads to increased satisfactions. However, interactivity can be viewed from a different dimensionality and edges. Interactivity is not only bounded to one particular issue. In our search, we uniquely view interactivity by how to let users have a complete control over the websites.

\section{Math and Equation}

Usability is the mainstay of any Web application that lead to user satisfaction and acceptance of the products. Our research is focusing on the usability of online social network sites that appears more robustly. Computer interfaces and applications are being mushroomed more robustly in the recent times. Optimized User Interface Design requires a systematic approach to the design process. But, to ensure optimum performance, Usability Testing and Evaluation are required. However, the usability of the online social network sites has been center-focus of quite number of researchers. For example, Steve Cornett examines the usability problems encounter by various levels of new players of massively multiplayer online role-playing games (MMORPGS). He identifies that most of the usability issues present a critical obstacles for players who lack of experience with genre [5]. This empirical testing and evaluation permit naïve users to provide data about what does work as anticipated and what does not work. Only after the resulting repairs are made can a product be deemed to have a user optimized interface. Arguable, the success experiments depend on the numbers of the participants involved; however, Virzi (1992) observes four or five participants will allow a usability practitioner to discover $80 \%$ of a product's usability problems [6]. Therefore, we can elicit that a few numbers of participant can represent a certain society or particular organizations.

\section{INTERACTIVITY FRAMEWORK}

Since the proliferation of online social network site is increasing dramatically, the usability evaluation of these social network sites needs accurate findings and investigation. Figure 2 is the procedure of our study to examine the usability of social network site through a real time mechanism for more efficient user experience. The selection methodology of social network site is based on the popularity attribute. We select Facebook and Twitter. These social network sites are quite popular [7]. Our study will require subjects who are varied in the level of familiarity with these sites. We involve participants from a different expertise to participate in our research. The contribution and sequence of actions of the participant will provide a clear identification to problems that encounter during the interaction of these two sites. In our methodology, the result of a sequence of user action will be gathered to discover and explore more usability flaws and to enhance the technology of web usability and the user experience. The interactivity framework is basically a procedure to entirely capture the sequence of user actions with the output of the conducted action. Interactivity framework primarily focuses on the user autonomy in taking control over the web navigations. To quantify the level of user satisfaction, we need to overly view the interactivity of the website by involving an actual user participating in the empirical research.



Fig. 2. Interactivity Framework Methodology.

During the participant engagement that is guided by a proposed framework, the participant is firstly asked to perform his/her intended action. Secondly, observation of the performed action is captured using the mentioned software in the next section. However, the interchangeable intended and observed action can be noticeably relative or otherwise. Sequentially, the result of actions might have been preserved an output in participant's intent. Observed output reflects the intended output of the participants. The accumulated observations of actions and outputs provide information or feedback about the web usability.

Interactivity and usability have relatively interchangeable relationship. Real time interaction between webs and its respective users determines the level of the usability. Without interaction with a specific products either tangible or intangible products, determination of the usability is not reliable and considered to be inconsistent. Interactivity with objects overly determines the usability. Arguably, evaluation of the interactivity is also a challenge task to predict or evaluate because it depends on the level of user expertise. Level of user's expertise can be hugely or slightly differ in interaction with a specific object from one user to another. 


\section{SOFTWARE USED}

We use Camtasia software [8], which is screen recording. In addition, it is a screen video capture. The presenter carries out all steps of the demonstration in sequence and is able to jump from one application to another without interrupting the recording process. Accordingly, this software records the interaction of the user while he/she engages in solving a problem. This software presents the performance of the experiments clearly. However, this software will help us to identify more flaws in the usability as well as the performance of the subjects in interacting with problems in the online social network sites. Figure 3 is a screenshot of the software capturing the participant interaction with a web site during the experiment.

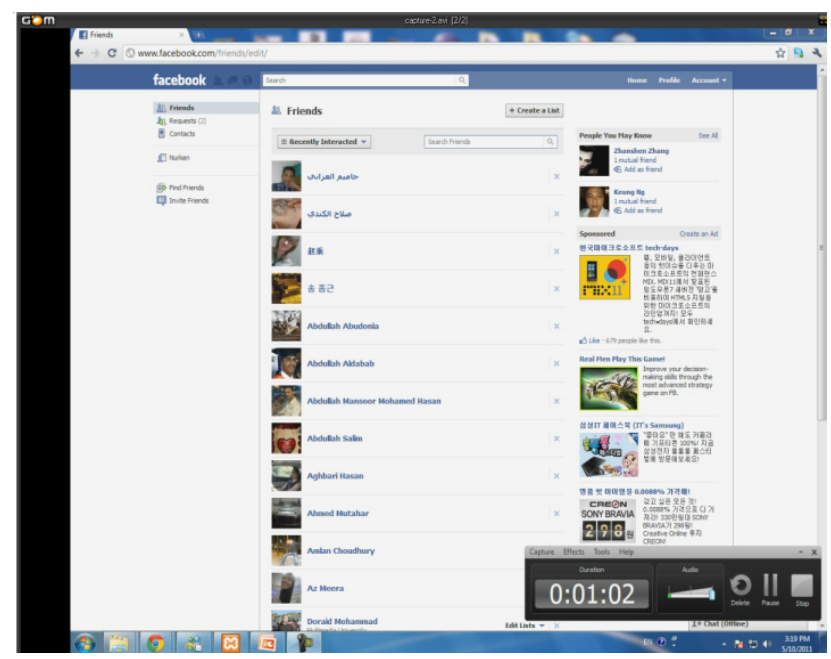

Fig. 3. Participant interaction using Camtasia software.

\section{TASK ANALYSIS}

The task of this study is to identify usability flaws and enhance the technology of web design for excellent user's experience. Based on the study framework, the task is to let the participant perform an intended action. Observation of the action will be captured by the aforementioned software. User action reveals the intended output. However, the user intended output may vary from the observed ones. The back and forth communication between the websites and its user is called interactivity. Every action is taken by a user must meet his/her intended result. If the result of the action fails to please the users, the consequence is frustration and anxiety to users. Moreover it causes the user to completely quite using websites. Therefore, the user action and its result determine the website stabilization and success. For instance, the user action is to retweet or post on someone's status or comments, the user expects to have unbounded text writing. In the next section, we will detail the research analysis and result based on the framework and the subject participation in our respective experiment. We will capture the participant contributions and feedbacks. Each and everything single feedback will have its significant impact on the enhancement of the web technology.

\section{RESEARCH ANALYSIS}

Previously, we mentioned the Camtasia software that captures the entire interaction and performance of participants. In our research five subjects were participated. They were expert and intermediate level in familiarity with social network website. We use Facebook and Twitter account to let the subjects have freedom in conducting his/her intended action. The actions were varied from one subject to another. We classified the actions of user based on the following attributes:

\section{Image viewers \\ 2. Comments \\ 3. Text area \\ 4. Privacy settings \\ 5. User profile}

As a result, multi-complaints were observed from user actions in regard to image viewer. The image viewer is interesting when it pops up without disturbing things that are running behind it, like loading video; however, if displaying the image takes longer time than expected and refreshing the image viewer is inevitable, the result of the action cause the image viewer to disappears and normal image viewer is showed up. Moreover, intended output from a refresh action is to refresh the image viewer without any transformation to other image viewer. In fact, the observed output causes frustration to the subjects especially when they are loading video at the back of the image viewer. Displaying image is annoying because sometimes it cause shaking up and down for the image viewer or the entire page. In addition, the action of rewinding previous and next image is irritated according to the subjects' feedback. For example, sometimes when the subjects click on the arrow that indicates the previous image display, the observed output is the next image.

In fact, comments are essential elements in our experiments. The actions of comments result in different observations and outputs. The main concern for the subjects, which they are varied in the level of familiarity, is when hitting the enter button, the intended output is to have a new line, however, the observed output is posting a comments instead. The consequence of the action was to remove the comment and rewrite the comment again. The subjects were not aware of the highlighted message under the text area, which tells the users be able to make a new line. As a result, the users wonder by telling "Why do I need to press shift + enter buttons to make new line 
instead of just one button?" therefore, we can conclude that, the consideration of these major feedbacks will give reduce flaws and give an exposure to new features of usability.

Text area is another characteristic of studying the interactivity. Text area is a place where the subject can write and comment. In the text area, major observations were noticeable from user actions. The subjects intended to write long text with some desirable format. However, the observed output was undesirable to the subjects. The text area is bounded to a few characters. Another observed output is when the subjects tried to find out a way that enables them to underline or bold specific text for another person to notice, they didn't find rich text feature provided. Limitations to number of characters causes the subjects narrow down their thoughts and constrain them to write another comments or status.

Privacy is the main issue that significantly concerns users. Users want to set their privacy that limited their information sharing. They need easily to configure their privacy according to their desires and preference. However, subjects in our empirical research found difficulties in navigating through the privacy settings. The settings were mysterious to the subjects. They could not fully understand the settings. For instance, the subjects were targeted to make friend list invisible to their friend, but the result of the action was vague. They couldn't accurately find what they are looking for. Moreover, the subjects were anxious when they found out that the output of their action was different from what they expected. User profile is the user representation on the site, which everyone else can be able to view the permitted shared information. However, according to our subject contribution in our research they identified that the profile pictures that appear underneath the basic information is undesirable. When they tried to hide the pictures, some find difficulties in identifying the feature that enable them to perform the desirable actions. Moreover, some of the subjects managed to identify the feature but when they tried it, they discovered the action button is unclickable. In comparison, Facebook outperforms Twitter in term of interactivity according to our empirical research. For instance, retweet text area is limited to less numbers of characters in Twitter compared to Facebook. However, commonly, the subjects contribution arose one common issue, which is retweet or recomment. Instead of quoting a person name as an indication of replying to his/her comments, alternative feature were pointed out. For example, to reply to one particular person or comments, text area or comment area should be underneath that particular user instead of the overlap comments and replies.

Figure 4 is a comparison between Twitter and Facebook in term of interactivity based on the subjects' feedback and evaluation. The evaluation is based on the interactivity framework. The result of the framework was effectively and empirically efficient in determining and identifying flaws of these two social network sites.

Moreover, the result and analysis of the research performed based on the interaction that was captured using the previous mentioned software. It helps us to study the interactivity of these web sites more profoundly.

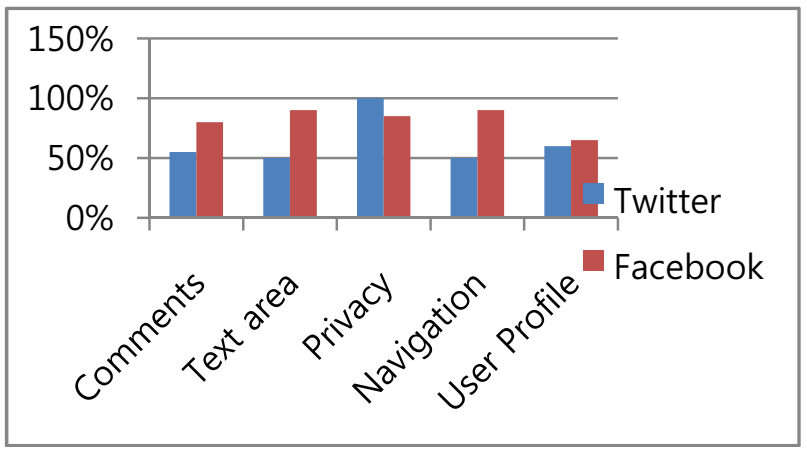

Fig. 4. Comparison between Facebook and Twitter

\section{IMPLICATION OF INTERACTIVITY FRAMEWORK ON USABILITY}

Interactivity framework is basically intended to enhance the usability of the web technology that evolves rapidly. It can be applied as guidelines for the web developer to enhance the usability. Effect of this framework can be diversified in many ways. We identify that the most critical problem is the structure of the web content. Structure and information architecture is vital in presenting the information for the users. Number of clicks should be minimally reduced. In comparison to other framework proposed by J.T. Mayes, C.J. Fowler [9], they proposed a framework that studies and evaluates the usability by extracting meaning from data, which undoubtedly the data is inconsistent and unreliable to be applied in the technology. However, in objection to the framework, the framework is not reliable in extracting data that does not involve real time interaction. Reliability of data needs actual user participation and interaction to determine and evaluate the usability of web.

TABLE I

COMPARISON BETWEEN INTERACTIVITY FRAMEWORK AND COURSEWARE FRAMEWORK[9]

\begin{tabular}{|l|rrrc|}
\hline attributes & Effective & efficient & reliable & Sufficient \\
\hline Interactivity & $\checkmark$ & $\checkmark$ & $\checkmark$ & $\checkmark$ \\
\hline Courseware & $\checkmark$ & $\checkmark$ & $\mathrm{x}$ & $\mathrm{x}$ \\
\hline
\end{tabular}




\section{CONCLUSION}

In this paper, we study the social network site interactivity to identify and avert usability problems for effective user's experience. The result of our research reveals significant issues. These issues need to be addressed to give an opportunity for the user to have self autonomy. Our methodology is effectively and empirically effective and efficient in identifying and averting usability issue in regard to social network sites, which it can vividly provide enhancement to the usability of the social network sites and the user experience.

\section{ACKNOWLEDGMENT}

This research was supported by 2010 Dongseo University research grants and Dongseo University's Ubiquitous Appliance Regional Innovation Center research grants from Ministry of Knowledge Economy of the Korean government.

\section{REFERENCES}

[1] D. Y. Wohn, et al., "The. "S." in social network games: initiating, maintaining, and enhancing relationships," Proc. 44th Hawaii International Conference on System Sciences (HICSS), pp. 1-10, Jan. 2011.

[2] J. Sung et al., "Social network game: exploring audience traits," Proc 28th international conference extended abstracts on Human factors in computing systems (CHI EA '10), pp. 3649-3654, April 2010.

[3] R. J. Stone. (August 2008). Human factors guidelines for interactive 3D and games-based training system design [Online]. Available: www.eece.bham.ac.uk/Default.aspx?tabid=154.

[4] R. R. Dholakia et al. (2000). Interactivity and revisits to websites: a theoretical framework, RITIM working paper. Available: http://ritim.cba.uri.edu/wp2001/wpdone3/interactivity.pdf.

[5] S. Cornett, "The usability of massively multiplayer online roleplaying games: designing for new users," Proc. SIGCHI conference on Human factors in computing systems (CHI '04), pp. 703-710, 2004.

[6] J. R. Lewis, "Sample sizes for usability studies: additional considerations," Human Factors: The Journal of the Human Factors and Ergonomics Society, vol. 36, no. 2, pp. 368-378, June 1994.

[7] E.-J. Lee. (June, 2010). What would life be like without social networking? [Online]. Available: http://joongangdaily.joins.com/article/view.asp?aid=2922318.

[8] TechSmith corporation. (2011). Screen recording and video editing software [Online]. Available: http://www.techsmith.com/camtasia/.

[9] J. T. Mayes and C. J. Fowler, "Learning technology and usability: a framework for understanding courseware," Interacting with Computers, vol. 11, no. 5, pp. 485-497, May 1999.

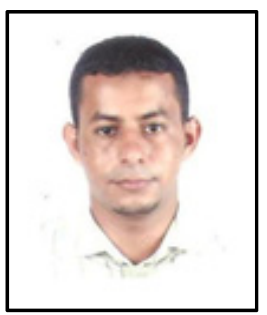

Sami Abduljalil is a Yemeni citizen who born on $1 / 2 / 1984$. He graduated as Bachelor Degree in Information System Egineering (Hons) Information technology in year 2010 at Multimedia Unversity, Cyberjaya, Malaysia (http://www.mmu.edu.my). He is currently also pursuing his master Degree in Ubiquitous Computing at Dongseo University (http://www.dongseo.ac.kr).

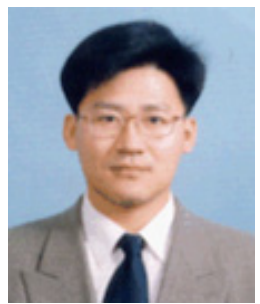

Gi-Hyun Hwang received his M.S. and Ph.D. in Electrical Engineering, Pusan National University. Recently, he is a Ph.D. He is currently a professor at Dongseo University. His research interested lies in applications of intelligent control to power system, RFID, and Embedded System. E-mail: hwanggh@gdsu.dongseo.ac.kr

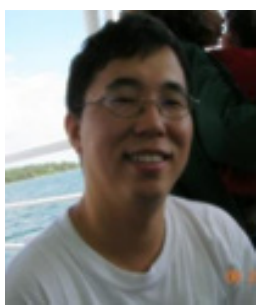

Dae-Ki Kang is an assistant professor at Dongseo University in South Korea. He was a senior member of engineering staff at the attached Institute of Electronics \& Telecommunications Research Institute in South Korea. He earned a $\mathrm{PhD}$ in computer science from Iowa State University in 2006. His research interests include social network services, machine learning, relational learning, statistical graphical models, metaheuristics, ontology learning, Tower of Hanoi, multimedia systems, intrusion detection, Web firewall, and computer vision. Prior to joining Iowa State, he worked at a Bay-area startup company and at Electronics and Telecommunication Research Institute in South Korea. He received a science master degree in computer science at Sogang University in 1994 and a bachelor of engineering (BE) degree in computer science and engineering at Hanyang University in 1992. 